\title{
A speckle-based CMOS sensor for arbitrary surface movement detection with correlated double sampling and gain error correction
}

\author{
Chao Wang*, Steve Tanner and Pierre-André Farine \\ Electronics and Signal Processing Laboratory (ESPLAB) \\ Ecole Polytechnique Fédérale de Lausanne (EPFL) \\ Rue Abram-Louis Breguet 2, 2000 Neuchatel, Switzerland
}

\begin{abstract}
We present a CMOS sensor for accurate tracking of speckle movements on arbitrary surfaces. The sensor is made of a pair of comb filters with a pitch of $5.6 \mu \mathrm{m}$ and decayed by $90^{\circ}$ to produce quadrature signals. The readout circuit is a $60 \mathrm{~dB}$ amplification chain with offset and KTC noise compensation. Integrated into a $180 \mathrm{~nm}$ CMOS process, the sensor and readout circuit occupy an area of about $0.1 \mathrm{~mm}^{2}$ and consume $24 \mu W$ at full speed of $64 \mathrm{ksample} / \mathrm{s}$. The direction and frequency of the quadrature signals are resolved externally by zero-crossing detection, giving an accuracy of about $5 \mu \mathrm{m}$. Thanks to a careful layout for gain error minimization, and the use of $K T C$ noise cancellation, a negligible residual drift was observed, and a minimal displacement of $5 \mu m$ was measured.
\end{abstract}

Keywords: CMOS photon sensor, gain error, correlated double sampling

\section{INTRODUCTION}

Spatial comb filtering (Figure 1) is a technique that performs a discrete spatial Fourier transform of the speckles image at one precise spatial frequency $\omega_{0}$. Motion detection by using the spatial comb filter was developed previously by Urban Schnell. ${ }^{1,2}$ A complete implementation ${ }^{3}$ based on CMOS technology was developed in our lab, where the motion detection device allowed a maximal stable tracking speed of the surface of $0.25 \mathrm{~m} / \mathrm{s}$ with a tracking accuracy of about $5 \mu \mathrm{m}$. However, this first implementation suffered from a limited speed tracking accuracy. In order to compensate for the sensor gain error, a differential readout sequence was used, which prevented correct operation of the correlated double sampling (CDS), resulting in a high system noise that affected significantly the accuracy at low speed motion tracking.

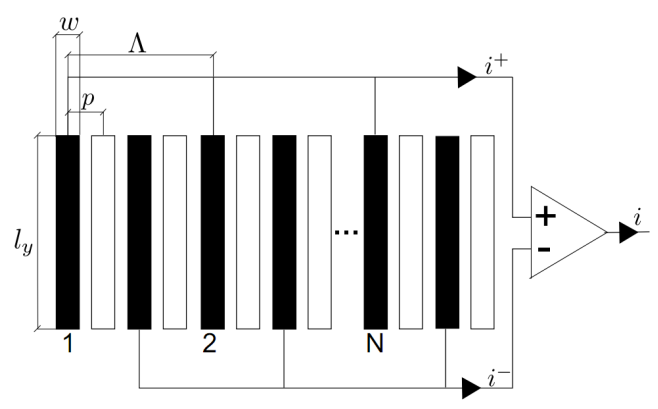

Figure 1. A comb filter array.

In this paper, a new sensor layout was proposed to correct for the gain error, while CDS was implemented to correct $\mathrm{kTC}$ noise.

*E-mail:chao.wang@epfl.ch, Telephone: +41 (0)32 7183433, http://esplab.epfl.ch/ 


\section{PHOTON SENSOR AND READOUT CIRCUIT DESIGN}

\subsection{Active pixel sensor (APS) and its operation}

A basic $\mathrm{APS}^{4}$ structure is drawn in Figure 2. A typical APS operation period has 3 phases:

1) Reset. Switching on the switch (PMOSFET), the photo-diode with a diode-capacitance $C_{p h}$ is initially charged with a specific bias reset voltage $V_{\text {reset }}$;

2) Integration. The illumination on the photo-diode causes the diode-capacitor to discharge proportionnally to the light intensity $L_{p h}$, resulting in a decreasing voltage $\Delta V_{p h}$ proportional to the photo-generated current $I_{p h}$ and the integration period $T$.

3) Readout. At the end of integration, the pixel voltage $V_{p h}$ is amplified by the readout circuit.
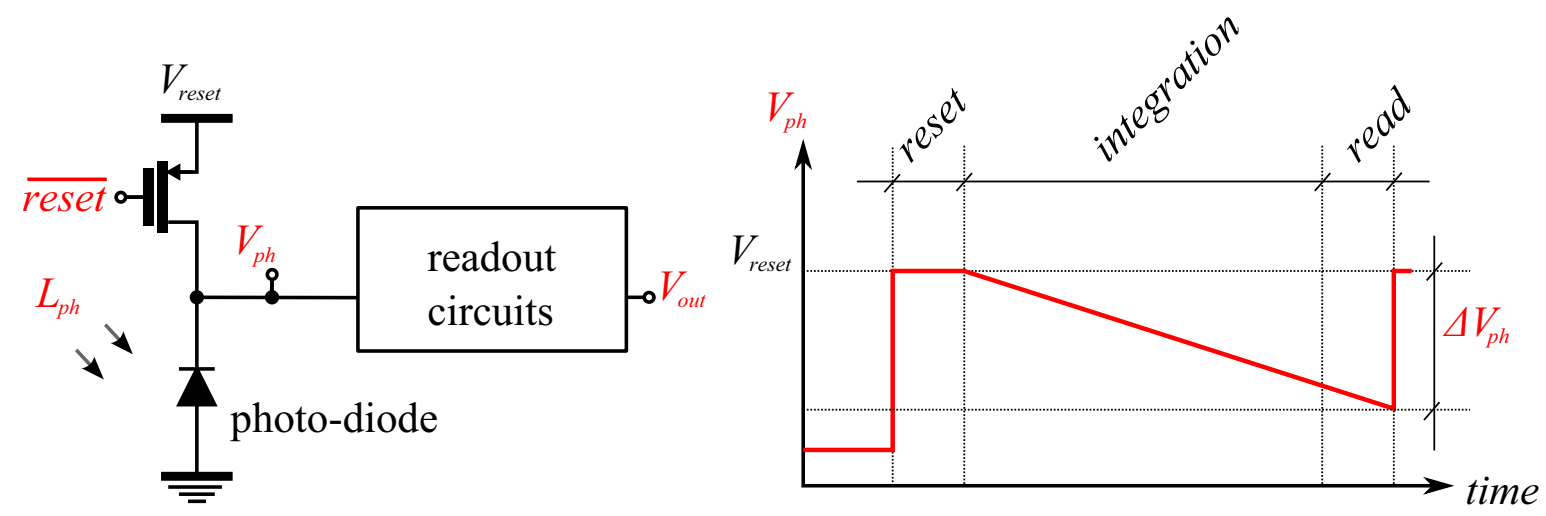

Figure 2. APS structure (left) and its operation phases (right).

As mentioned in our previous paper, ${ }^{3}$ for a given optical power, the quantum efficiency (QE), which includes the fill-factor, determines the sensor output signal. If we define the light intensity (number of photons arriving on the sensor surface per second and per surface unit) as $L_{p h}$, we have:

$$
I_{p h}=Q E \cdot L_{p h} \quad \Delta V_{p h}=I_{p h} C_{p h} \cdot T .
$$

\subsection{Readout circuit}

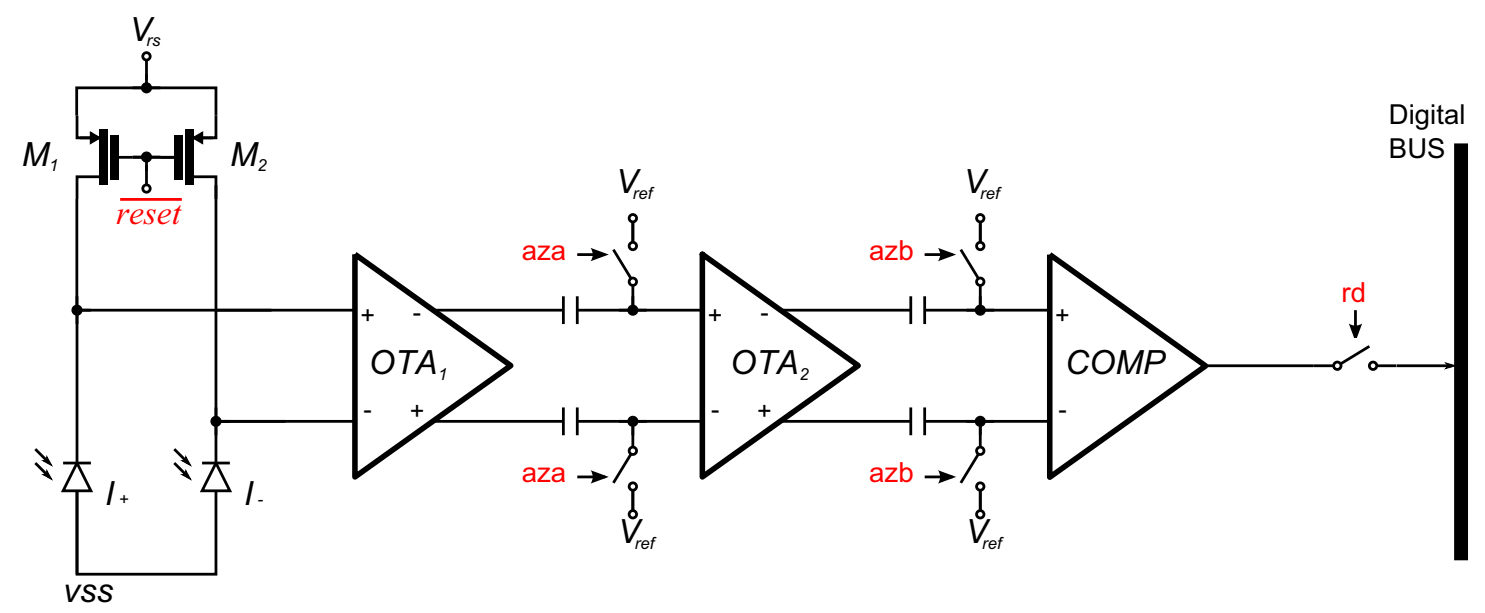

Figure 3. Readout circuit with amplification chain and offset correction. 
In order to perform a spatial comb filter, the imaginary part (sine) and real part (cosine) functions are implemented with 4 interlaced comb photo-detector arrays connected differentially. The differential signal is amplified by the readout circuit, made of two cascaded OTAs with an overal gain of $60 \mathrm{~dB}$ and a voltage comparator at the end. Figure 3 shows the structure of the readout circuit.

Compare to our previous design, the following optimizations were done:

1) The input voltage range of OTA1 and OTA2 being small, the common mode feedback was not implemented. For the single-stage OTA, we introduced a cross-coupled active load in parallel to the diode-connected load. The structure shown in Figure 4. The cross-coupled transistors $M_{N 4}$ and $M_{N 5}$ create a negative differential resistance, which acts so as to increase the resistance of the load formed by $M_{N 6}$ and $M_{N 7}$. If their difference is small, the OTA gain can be boosted to a high value. In order to minimize hysteresis and guarantee system stability, the difference between positive and negative resistances is kept significant, resulting into a limited OTA gain. As this structure does not have a common mode feedback, it saves more than half of the power consumption compared to the previous design.

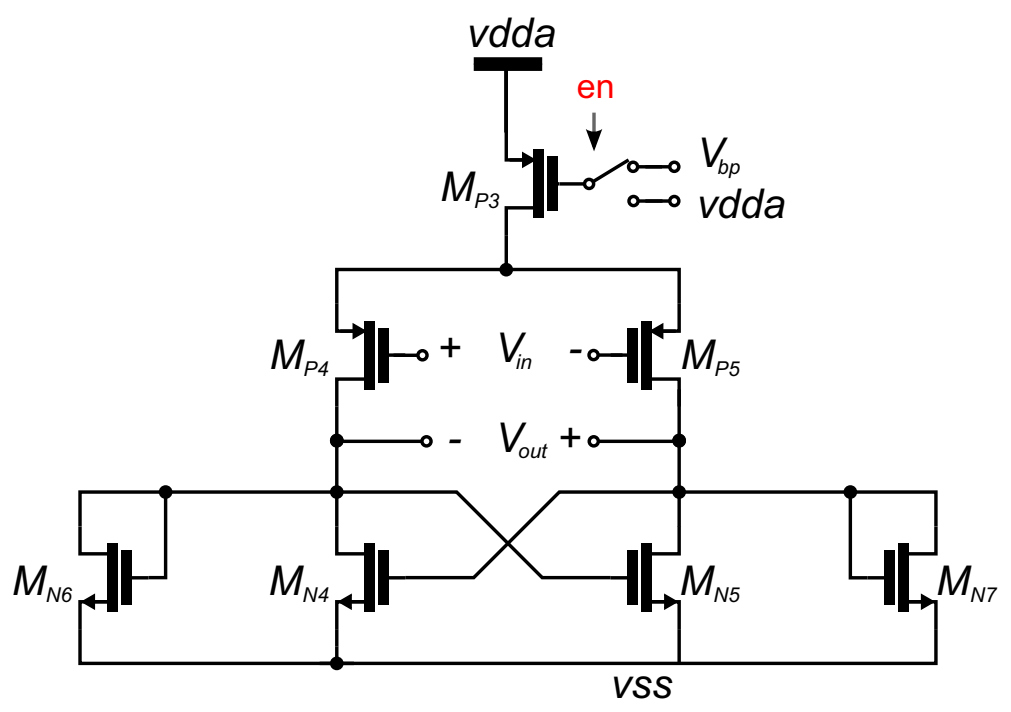

Figure 4. Cross coupling structure of OTA1 and OTA2.

2) In the previous work, ${ }^{3}$ a 3 -bit ADC was terminating the readout electronics. Since it appeared that motion detection implemented with zero-crossing was working fine, and was requiring a single comparator, the 3-bit ADC was removed.

3) The gain error is optimized with a careful sensor layout which will be presented later on.

\section{OFFSET ERROR AND ITS COMPENSATION}

The offset error $O$ is defined as constant error voltage added to the photo-diode voltage $V_{p h}$. It is caused by 1 ) charge injection in the reset transistors, 2) transistor mismatch in the amplification path, and 3) kTC noise of the pixel reset operation.

In fact, KTC noise is not an offset but the thermal noise of the reset transistor, sampled by the photodiode capacitor when the reset switch opens. After sampling, this noise stays constant and can be considered as an offset error, and is corrected the same way than the other offset contributions.

The typical method to compensate for offset is the Correlated Double Sampling(CDS) or auto-zero ${ }^{5}$ operation. It is implemented by adding storage capacitors between the OTA stages, which are used to store the amplifier outputs at the end of the reset operation, thus storing at the same time all the offset sources. For this, switches $a z a$ and $a z b$ connect the capacitors and OTA inputs to the zero voltage reference $V_{r e f}$. At the end of the integration phase, the readout circuit amplifies only the voltage difference before and after integration. 


\section{GAIN ERRORS AND ITS OPTIMIZATION}

Gain error is the difference of sensitivity between the positive and negative sensors. It is signal-depended. Because of the low contrast of speckles, even a small gain error can render the movement detection impossible. In such a miniature optical system, the main sources of gain error are mismatch and gradient in doping concentration, and illumination non-homogeneity. The typical way to compensate for technology gradients is to use a common centroid layout, but due to the comb filter structure, common centroid symmetry between negative and positive paths is not easily achieved. In this design, we propose some optimizations in sensor layout to cancel the gain error.

\subsection{Gain error correction}

1) Layout with common centroid symmetry. In this new layout, 8 fingers instead of 4 are ranged in one spatial period. Let us consider the real part signal $i$, as example, shown in Figure 5. One negative and one positive finger are replaced by two negative and two positive fingers, respectively. Placing the very right finger to the very left, common centroid symmetry between positive and negative paths can be achieved. Since the previous design used the minimal dimension that is allowed from silicon technology for the finger width and neighbor distance, the spatial period in this new layout is doubled.

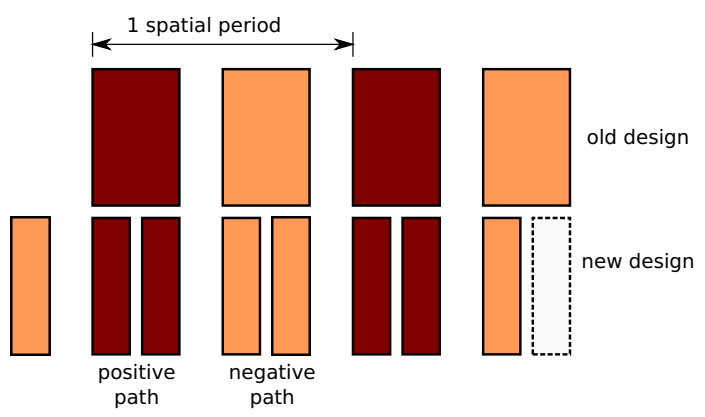

Figure 5. Layout with common centroid symmetry.

2) Removed metal tracks between sensor fingers. Metal tracks between fingers were placed to increase illumination contrast. Since metal masks also reduce the quantum efficiency (QE) and add additional capacity to the photo-diode capacitor, they were removed.

3) Added dummy fingers at both side of each comb filter array. This optimization will make every finger approach the same electrical environment.

\subsection{New sensor layout after gain error optimization}

The entire sensor array with all the optimization is showed in Figure 6.8 pixel fingers with the same color form half of a comb filter, named as ip in $q p$ and $q n$. The basic dimension and electrical parameters are summarized in Table 1 . 


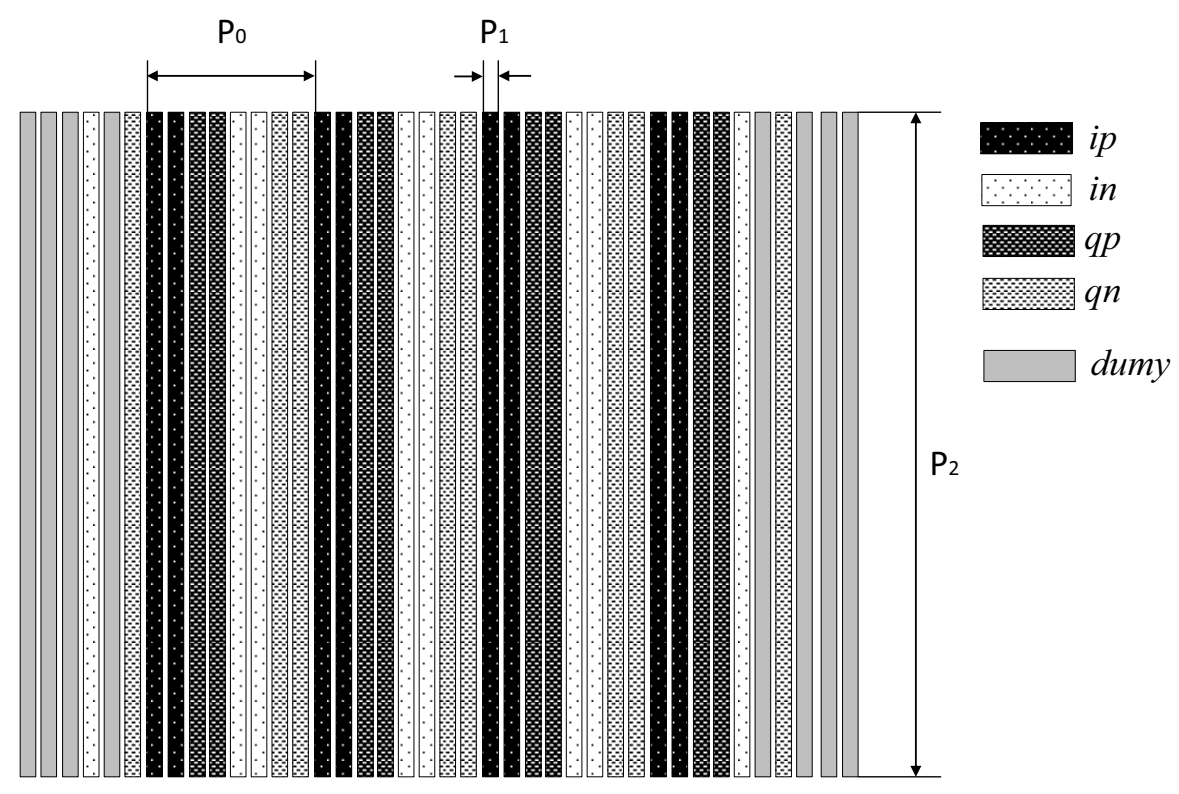

Figure 6. Signal, error and noise analysis of APS.

Table 1. Basic sensor size parameters.

\begin{tabular}{|l|l|l|}
\hline Parameter & Value & Unit \\
\hline Total finger number & 40 & - \\
\hline Finger number for a comb filter & 8 & - \\
\hline Dumb finger number & 8 & - \\
\hline spatial period p0 & 22.4 & $\mu m$ \\
\hline pitch size p0/8 & 2.8 & $\mu m$ \\
\hline Photodiode (nwell) width p1 & 1 & $\mu m$ \\
\hline Photodiode (nwell) length p2 & 90 & $\mu m$ \\
\hline Photodiode capacitance $C_{\text {finger }}$ (1 finger) & 180 & $f F$ \\
\hline
\end{tabular}

\section{FABRICATION AND TESTS}

\subsection{Electrical system}

The chip including the sensors and analog circuits was fabricated into a $180 \mathrm{~nm}$ CMOS process. It was bonded on a PCB test board. The digital timing and controlling signals generated by a FPGA board are sent to the chip. The digital outputs $i$ and $q$ signals from the chip are sent back to the FPGA. The motion detection algorithm is processed in the FPGA. The final motion vectors are printed on the FPGA development board display*, showing also the accumulated motion.

\subsection{Optical system}

An optical system, shown in Figure 7, is built to generate speckles with the size matching the comb filter. The laser (wavelength of $\lambda$ ) is generated from a laser emitter at the focal point of lens (the lens focal length is $f$ ),

\footnotetext{
${ }^{*}$ Nexys ${ }^{T M} 3$ Spartan-6 FPGA Board
} 
therefore a collimated beam is formed. After the aperture, the spot with diameter $D$ is projected on a speckle diffuser which is a rough translucent sheet. The chip with photo-sensor keeps a distance $z$ away from the diffuser. The speckles image is then formed on the sensor surface.

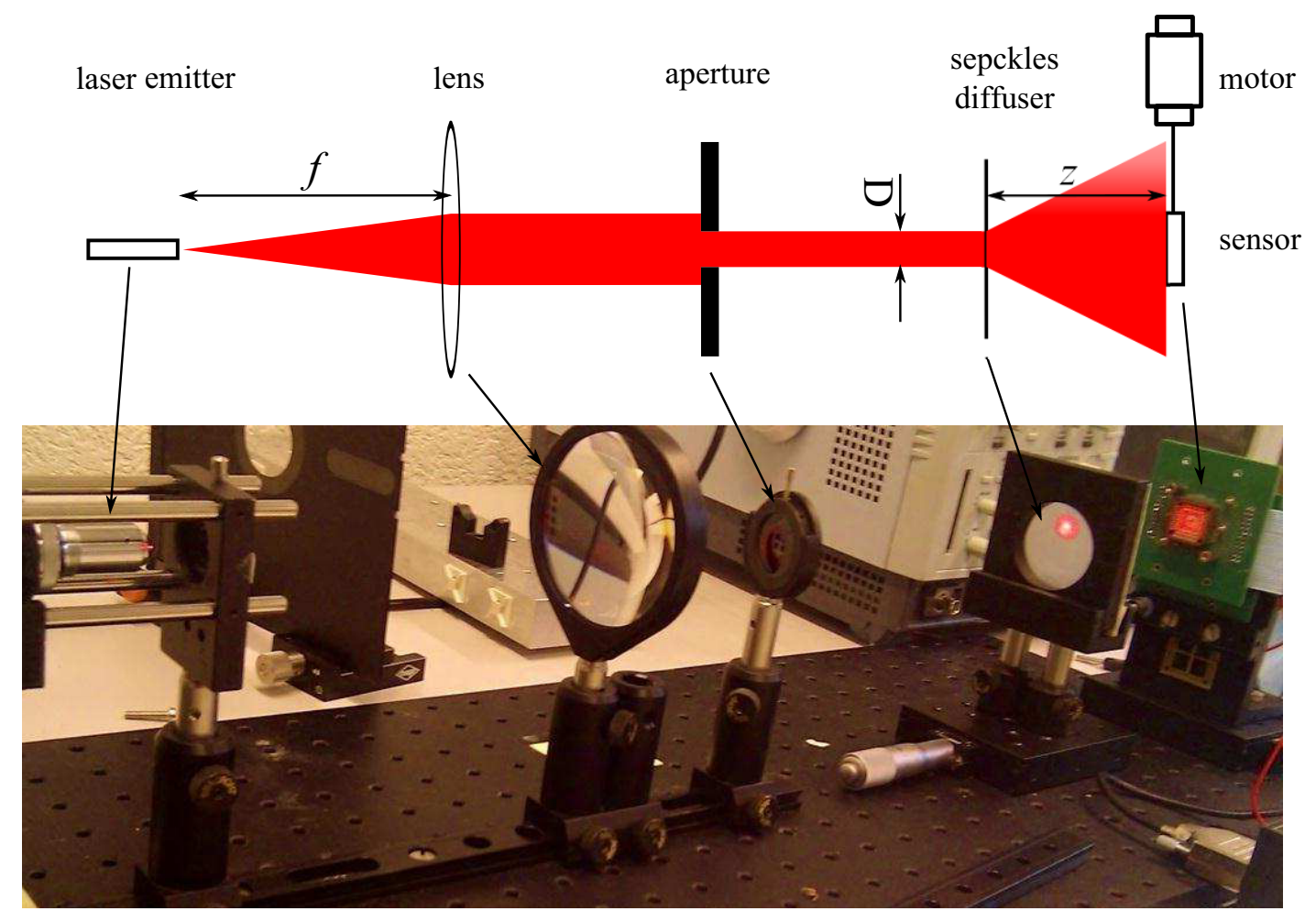

Figure 7. Optical setup for testing.

From the paper of Urban Schnell, ${ }^{1}$ the speckle size is given by:

$$
\Delta x_{s}=\frac{4}{\pi} \frac{\lambda z}{D}
$$

For such speckle size, the needed spatial period $\Lambda$ of the comb photo-detector array is: ${ }^{6}$

$$
\Lambda=\frac{\Delta x_{s} \pi}{2} .
$$

For our setup, $\Lambda=22.4 \mu \mathrm{m}, D=10.79 \mathrm{~mm}, z=30 \mathrm{~cm}, f=10 \mathrm{~cm}$ and $\lambda=632.8 \mathrm{~nm}$.

\subsection{Testing methods}

Compared to the previous design, this design has been improved in gain error correction and offset error compensation. The test should prove that the system has a better performance on low speed tracking which was poor in the previous design. The test method should also be able to repeat a number of times in order to have a statistical result.

We choose a micro-motion motor system ${ }^{\dagger}$ to control the sensor movement. The PCB with the sensor is fixed on the translation stage which is monitored by the motor. The motor is able to move the sensor repeatedly in one direction back and forth with an accuracy of $29 \mathrm{~nm}$ and a stable speed. The acceleration and deceleration can be easily controlled. With the recorded stop point positions in the back-and-forth movement, we can measure the repeatability and precision of the motion tracking system.

\footnotetext{
${ }^{\dagger}$ THORLABS Z825B
} 


\subsection{Test results}

126 times of continuing $1000 \mu \mathrm{m}$ back-and-forth movements were recorded. The speed of $0.1 \mathrm{~mm} / \mathrm{s}$ is carefully controlled by the motor, the acceleration and deceleration near stop points are fixed to $1 \mathrm{~mm} / \mathrm{s}^{2}$. Figure 8 shows the recorded stop point positions. The average of detected movements (back and forth) is $998.056 \mu \mathrm{m}$ and the standard deviation is $2.8964 \mu \mathrm{m}$. The average repeatability ${ }^{\ddagger}$ is calculated to be $99.71 \%$ and the precision ${ }^{\S}$ is up to $99.81 \%$.

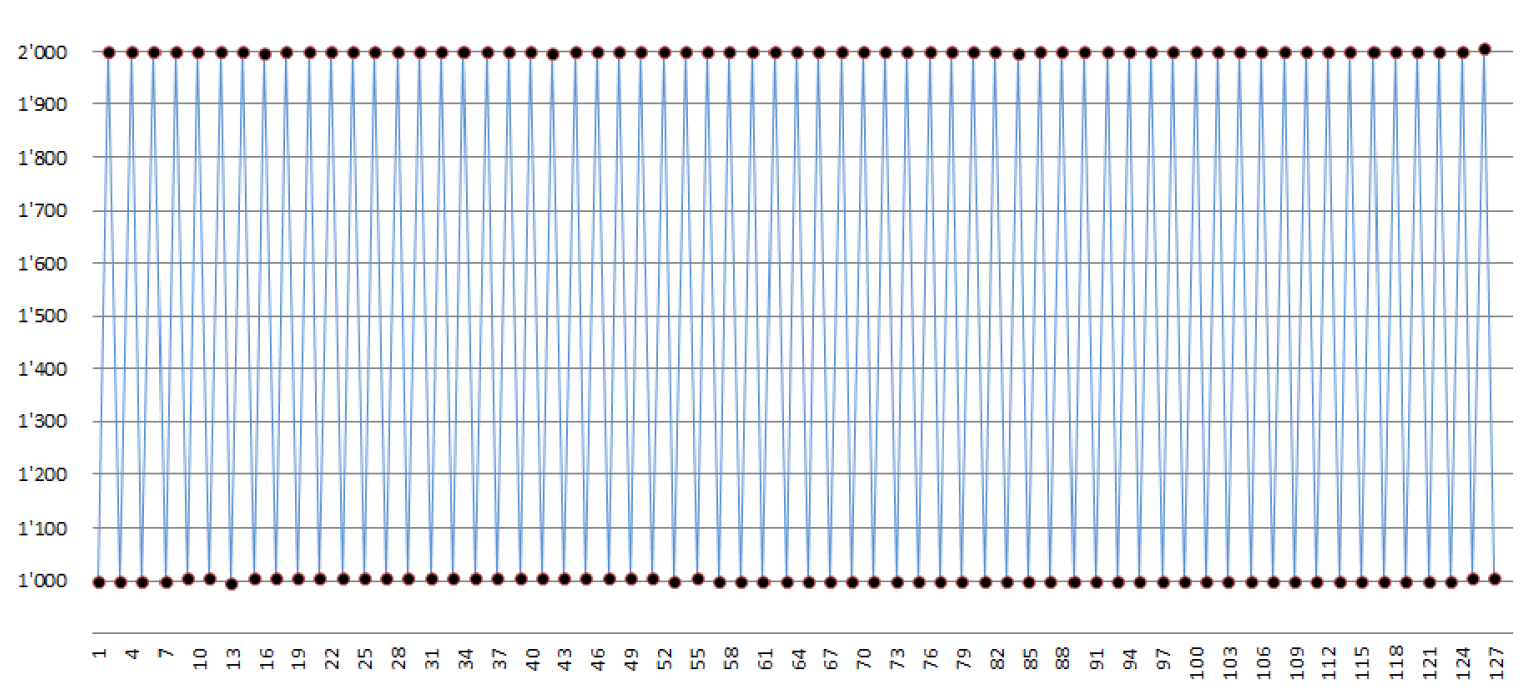

Figure 8. Repeated moving test: 126 times of moving back and force between position $1000 \mu \mathrm{m}$ to $2000 \mu \mathrm{m}$ with the speed of $0.1 \mu \mathrm{m} / \mathrm{s}$. The horizontal axis is the recoding sequence, and the vertical shows the position(in unit of $\mu \mathrm{m}$ ).

In these tests, the main source which affects the precision is the system mechanical noise. Due to the limited comb filter pitch, the minimum detectable displacement is $5.6 \mu \mathrm{m}$. The movement distance which is shorter than $5.6 \mu \mathrm{m}$ will create a noisy result when the movement is resolved with zero-crossing detection. By using 8 sensors working in parallel like in the previous design, and taking the average as detected motion, it is possible to reach better repeatability and accuracy.

Other tests show that the tracking system allows a maximal tracking speed of $0.25 \mathrm{~m} / \mathrm{s}$. The most serious drift problem in our previous design ${ }^{3}$ does not appear after the optimization.

These results show that an optimization of the low speed tracking performance could be achieved without reducing the high speed performance.

\section{CONCLUSION}

A CMOS sensor system for the detection of speckle movements was designed, fabricated and tested. The sensor is made of a pair of comb filters with a pitch of $5.6 \mu \mathrm{m}$ and decayed by $90^{\circ}$ to produce quadrature signals. Common centroid symmetrical layout and some other optimization have been implemented to corrected the gain error. Correlated double sampling (CDS) operation compensates for the offset error and the KTC noise. Integrated into a $180 \mathrm{~nm}$ CMOS process, the sensor and readout circuit occupy an area of about $0.1 \mathrm{~mm}^{2}$ and consume $24 \mu \mathrm{W}$ at full speed of $64 \mathrm{ksample} / \mathrm{s}$. The motion detection algorithm is resolved by zero-crossing detection on an FPGA board. No residual drift was obsedved. The result shows good performance both at high and low speed tracking.

\footnotetext{
${ }^{\ddagger}$ repeatability $=1$ - standard deviation / mean

${ }^{\S}$ precision $=$ tested result $/$ reference value
} 


\section{ACKNOWLEDGMENTS}

We thank the swiss Commission for Technology and Innovation (CTI) for funding. We thank also our colleagues of the Optics and Photonics Technology Lab (OPTLab) and the Laboratory of Microengineering for Manufacturing (LPM) of EPFL.

\section{REFERENCES}

[1] U. Schnell, J. Piot, and R. Dandliker, "Detection of movement with laser speckle patterns: statistical properties," Journal of the Optical Society of America a-Optics Image Science and Vision 15(1), pp. 207-216, 1998.

[2] U. Schnell, R. Dandliker, and S. Gray, "Dispersive white-light interferometry for absolute distance measurement with dielectric multilayer systems on the target," Optics Letters 21(7), pp. 528-530, 1996.

[3] C. Wang, S. Tanner, and P. Farine, "A speckle-based cmos sensor array for arbitrary surface movement detection," in Integrated Photonics: Materials, Devices, and Applications, A. L. Ali Serpenguel, Giancarlo C. Righini, ed., Proceedings of SPIE 8069, 2011.

[4] E. R. Fossum, "Cmos image sensors: Electronic camera-on-a-chip," Ieee Transactions on Electron Devices 44(10), pp. 1689-1698, 1997.

[5] R. Poujois and J. Borel, "Low drift fully integrated mosfet operational-amplifier," IEEE Journal of SolidState Circuits 13(4), pp. 499-503, 1978.

[6] E. Archbold and A. Ennos, "Displacement measurement from double-exposure laser photographs," Optica Acta: International Journal of Optics 19(4), pp. 253-271, 1972. 